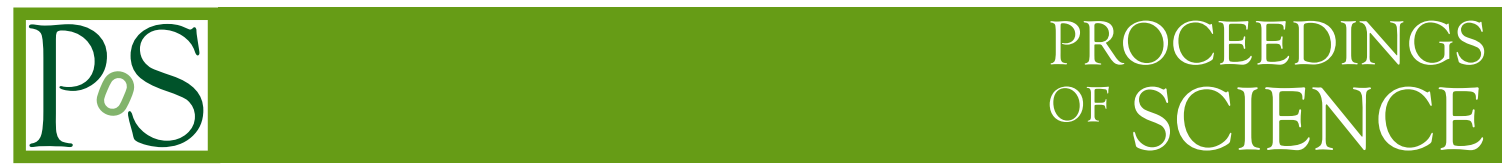

\title{
Charm-Hadron Production at Hadron Colliders
}

\author{
Miriam Watson*, on behalf of the LHC and Tevatron experimental collaborations \\ University of Birmingham (GB) \\ E-mail: miriam,watsonecern.ch
}

\begin{abstract}
Recent results on charm hadron production are presented, using data recorded in proton-proton collisions at the Large Hadron Collider and in proton-antiproton collisions at the Tevatron. These results include the production of charmonium and of open charm mesons, and their comparison with theoretical predictions. Measurements of the associated production of hidden or open charm mesons with additional quarkonium states are also presented.
\end{abstract}

38th International Conference on High Energy Physics

3-10 August 2016

Chicago, USA

${ }^{*}$ Speaker. 


\section{Introduction}

The production of heavy quarks at hadron colliders allows the predictions of quantum chromodynamics (QCD) to be tested, and gives vital information on the modelling of signal and background processes in searches for new physics beyond the Standard Model. This report covers recent results on charm hadron production at the Large Hadron Collider (LHC) and the Tevatron. These measurements fall into three categories: charmonium production at the LHC, open charm properties from the Tevatron and the LHC, and several results on associated production of charm hadrons with additional quarkonium states. Results will be presented from the ATLAS, CMS, LHCb and ALICE collaborations at the LHC, and from the CDF and D0 experiments at the Tevatron.

\section{Charmonium properties}

The charmonium states discussed here $(J / \psi$ and $\psi(2 S))$ are reconstructed in their di-muon final states, $\mu^{+} \mu^{-}$. These are easy to identify in the detectors and can be recorded using dedicated di-muon triggers. Measurements have been made for prompt and non-prompt signatures, where 'prompt' charmonia are those produced in the primary interaction or through feed-down from higher states, and 'non-prompt' are those originating from the decays of B-hadrons. The two components are usually separated by a combined fit to the invariant mass and the decay time (or decay length). Prompt mesons decay close to the primary event vertex, while non-prompt particles typically have a longer lifetime.

ATLAS [1] has measured double-differential cross-sections as a function of transverse momentum $p_{\mathrm{T}}$ and rapidity $y$, for $J / \psi$ and $\psi(2 \mathrm{~S})$ mesons in the range $8<p_{\mathrm{T}} \leq 110 \mathrm{GeV}$ and $|y|<2$, at centre-of-mass energies of both 7 and $8 \mathrm{TeV}$ [2]. As an example, the prompt $J / \psi$ cross-section at $7 \mathrm{TeV}$ is shown in Fig. 1, compared to the NLO calculation NRQCD [3]. This prediction includes colour singlet and octet contributions, together with long-distance matrix elements tuned to earlier experimental data, and shows a fair agreement with the data. The non-prompt cross-sections are compared to the fixed-order-next-to-leading log predictions, FONLL [4]. The theory describes the shape well but gives higher yields for $\psi(2 \mathrm{~S})$; in contrast, it predicts a slightly harder $p_{\mathrm{T}}$ spectrum for $J / \psi$ than in the data. ALICE has also measured $\psi(2 \mathrm{~S})$ and $J / \psi$ production at $8 \mathrm{TeV}$ [5], in the forward rapidity region $2.5<y<4$. The differential cross-sections are measured down to zero $p_{\mathrm{T}}$, and the $J / \psi$ distribution agrees reasonably well with a previous LHCb measurement [6] (differing by $<1.7 \sigma$ at low $p_{\mathrm{T}}$ and low $\left.y\right)$. The inclusive ratio of $\psi(2 \mathrm{~S})$ to $J / \psi$ cross-sections is measured as $0.14 \pm 0.01$ (stat.) \pm 0.02 (syst.) and is consistent with earlier results at $7 \mathrm{TeV}$.

CMS has preliminary results which extend the centre-of-mass energy to $13 \mathrm{TeV}$, using the 2015 data [7]. The double-differential cross-sections are measured for $J / \psi$ and $\psi(2 S)$ for $p_{\mathrm{T}}$ values up to 120 and $100 \mathrm{GeV}$, respectively, for $|y|<1.2$. Different rapidity slices show consistent behaviour as a function of $p_{\mathrm{T}}$. Fig. 2 shows the $J / \psi$ and $\psi(2 \mathrm{~S})$ differential cross-sections, compared to those measured at $7 \mathrm{TeV}$. The ratio plot shows that the cross-section at $13 \mathrm{TeV}$ is 2 to 3 times higher than at $7 \mathrm{TeV}$, and increases slowly as a function of $p_{\mathrm{T}}$. This behaviour is consistent with the expected evolution of the parton density functions.

Also at $13 \mathrm{TeV}, \mathrm{LHCb}$ has measured $J / \psi$ production in the forward region, for $2<y<4.5$ and $p_{\mathrm{T}}<14 \mathrm{GeV}$ [8]. The prompt $J / \psi$ differential cross-section is compared to NRQCD predictions, 


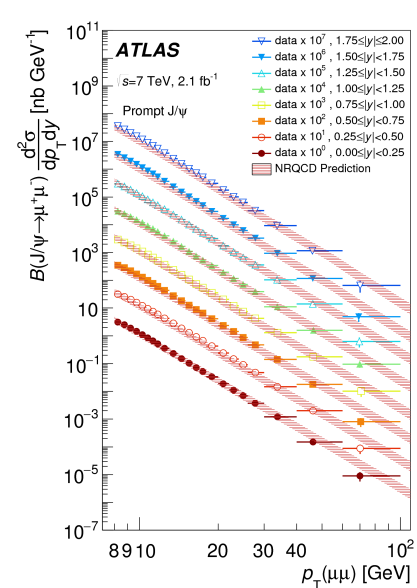

Figure 1: ATLAS prompt $J / \psi$ differential cross-section as a function of $p_{\mathrm{T}}$ at $7 \mathrm{TeV}[2]$.
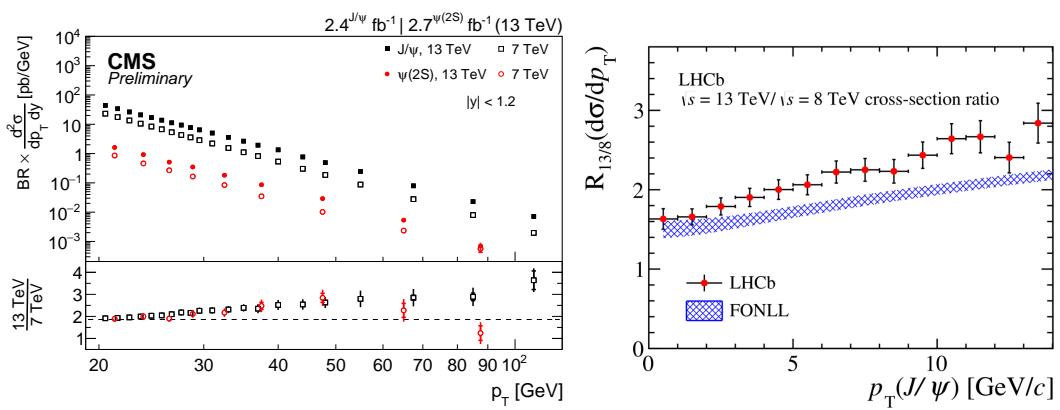

Figure 2: CMS $\psi(n S)$ differential cross sections as a function of $p_{\mathrm{T}}$ at $7 \mathrm{TeV}$ and $13 \mathrm{TeV}$ [7].
Figure 3: LHCb ratio of nonprompt $J / \psi$ differential crosssections at $13 \mathrm{TeV}$ and $8 \mathrm{TeV}$ [8].

and the non-prompt data are compared to FONLL. Both calculations agree with data within the uncertainties and within their range of validity. The same predictions are compared to the ratio of $13 \mathrm{TeV}$ to $8 \mathrm{TeV}$ production. In this case, the NRQCD prediction agrees reasonably well with the prompt ratio, while the FONLL non-prompt prediction lies some way below the measurements (Fig. 3), even when the theoretical uncertainties are taken into account.

Each of these measurements allows the non-prompt fraction to be determined, where this is given by the ratio of non-prompt charmonium to the inclusive (prompt and non-prompt) sample. The measurements at central rapidities, from ATLAS [9] and CMS [7], are presented for a variety of centre-of-mass energies ranging from 2.76 to $13 \mathrm{TeV}$, and are also compared with a CDF result at $1.96 \mathrm{TeV}$. The non-prompt fraction is found to increase with $p_{\mathrm{T}}$, but has little dependence on rapidity. It shows a slight increase relative to measurements at lower energies. The LHCb nonprompt fraction for forward rapidities also increases with $p_{\mathrm{T}}$, but is found to decrease as the rapidity increases [8].

\section{Open charm production}

Moving from the hidden charm states to open charm, the LHC and Tevatron experiments have measured the properties of a number of $D$ mesons. These are produced in the decay chains of both charm and bottom quarks, and can be calculated to next-to-leading order. There are still considerable theoretical uncertainties, and understanding these states is important for the backgrounds to the Higgs boson and new physics channels. All the measurements are made using hadronic decay modes, in the channels $D^{0} \rightarrow K^{-} \pi^{+}, D^{+} \rightarrow K^{-} \pi^{+} \pi^{+}, D_{s}^{+} \rightarrow \phi \pi^{+} \rightarrow\left(K^{-} K^{+}\right) \pi^{+}$, $D^{*+} \rightarrow D^{0} \pi^{+} \rightarrow\left(K^{-} \pi^{+}\right) \pi^{+}$and their charge conjugates.

A recent analysis from CDF measures $D^{+}$production for $p_{\mathrm{T}}>1.5 \mathrm{GeV}$ and $|y|<1$ in protonantiproton collisions at $1.96 \mathrm{TeV}$ [10]. The signal is separated from background using a simultaneous fit to the $K \pi \pi$ invariant mass and the $D^{+}$transverse impact parameter relative to the primary vertex. The total cross-section within the fiducial region is $71.9 \pm 6.8$ (stat.) \pm 9.3 (syst.) $\mu \mathrm{b}$, and the 
$p_{\mathrm{T}}$ spectrum is compared with the FONLL prediction in Fig. 4. Data and theory are seen to agree within uncertainties, but show an overall difference in shape.

ATLAS has measured $D^{*+}, D^{+}$and $D_{s}^{+}$mesons with the $7 \mathrm{TeV}$ proton-proton data [11]. Fiducial cross-sections have been determined for all three particles, and differential cross-sections for $D^{*+}$ and $D^{+}$. These are compared with a variety of next-to-leading-order predictions in a $p_{\mathrm{T}}$ region extending up to $100 \mathrm{GeV}$. The GM-VFNS [12] calculation gives the best description of data in shape and normalisation, while FONLL, POWHEG [13] and MC@NLO [14] agree with data within uncertainties. After extrapolation to the full phase space, the total $c \bar{c}$ cross-section is measured to be $8.6 \pm 0.3$ (stat.) \pm 0.7 (syst.) \pm 0.3 (lum.) \pm 0.2 (ff) $\pm_{-3.4}^{+3.8}$ (extr.) mb, where the last three uncertainties are from the luminosity, fragmentation fraction and extrapolation uncertainties, respectively.

LHCb has presented differential cross-sections for $D^{0}, D^{+}, D_{s}^{+}$and $D^{*+}$ mesons in the $13 \mathrm{TeV}$ proton-proton data [15]. The measurements agree with POWHEG, FONLL and GM-VFNS nextto-leading order calculations within uncertainties, but tend to lie above the predictions. When compared to the ratio of 13 to $7 \mathrm{TeV}$ differential cross-sections, the predictions are consistently lower than the data. The $c \bar{c}$ cross-section is measured to be $2840 \pm 3$ (stat.) \pm 170 (syst.) \pm 150 (ff) $\mu \mathrm{b}$ within the fiducial volume $p_{\mathrm{T}}<8 \mathrm{GeV}$ and $2.0<y<4.5$, and is compared to predictions in Fig. 5.

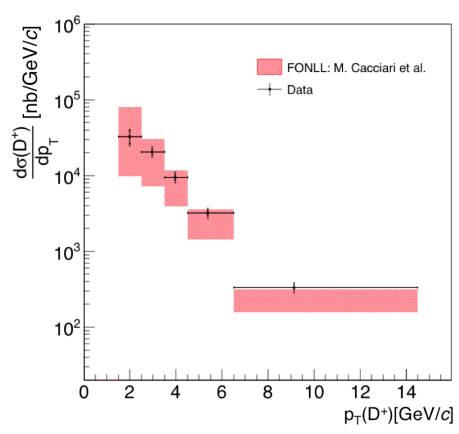

Figure 4: $\mathrm{CDF} \mathrm{D}^{+}$differential cross-section as a function of $p_{\mathrm{T}}$ at $1.96 \mathrm{TeV}$ [10].

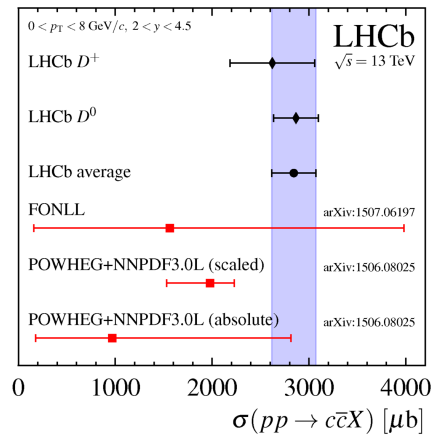

Figure 5: $\mathrm{LHCb} c \bar{c}$ fiducial cross-section at $13 \mathrm{TeV}$ [15].

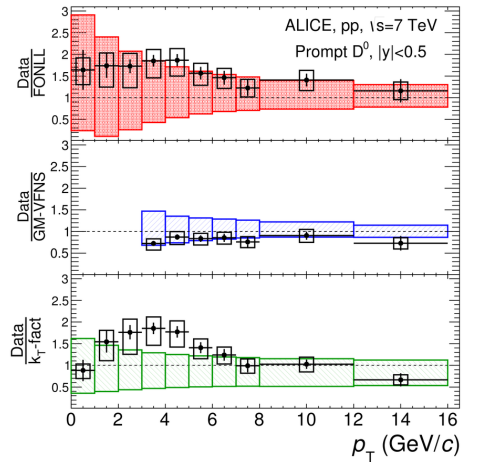

Figure 6: ALICE $\mathrm{D}^{0} p_{\mathrm{T}^{-}}$ differential cross-section at $7 \mathrm{TeV}$, relative to theory [16].

ALICE has performed an analysis of $D$ meson production in proton-proton collisions at $7 \mathrm{TeV}$ and proton-lead collisions at $5 \mathrm{TeV}$ [16]. Two methods are combined for the $D^{0}$ measurement, to extend the measurement down to a $p_{\mathrm{T}}$ of zero. At higher $p_{\mathrm{T}}$, the $D^{0}$ candidates are identified with track vertexing, while at low $p_{\mathrm{T}}$ the signal is extracted using a variety of background estimates, such as event mixing or sideband fits. The comparison with models in Fig. 6 shows general agreement within uncertainties, although the central values of FONLL lie below the data, GM-VFNS lies above, and the leading order $k_{T}$ factorisation model [17] varies with $p_{\mathrm{T}}$. The total $c \bar{c}$ cross-section is determined to be $8.18 \pm 0.67$ (stat.) \pm 0.90 (syst.) ${ }_{-0.36}^{+2.40}$ (extr.) \pm 0.29 (lumi.) \pm 0.36 (ff) mb.

There are recent $D^{0}$ measurements from CMS [18] and LHCb [19], comparing the protonproton, proton-lead and lead-lead environments. These have been covered in the Heavy Ion contributions to this conference. Two new ALICE measurements look at the kinematics of $D$ meson production in more detail. In the first, the $D$ meson yields are evaluated as a function of charged particle multiplicity in the central region in proton-lead events [20]. The particle yields show a 
faster than linear increase with multiplicity, which is independent of the $p_{\mathrm{T}}$ of the $D$ meson. In the second analysis, the azimuthal dependence of hadronic activity relative to the $D$ meson is compared for proton-proton and proton-lead data [21].

\section{Associated production of charm hadrons}

The last category of charm hadron measurements is the associated production of charm hadrons with quarkonia. LHCb has observed (at more than $5 \sigma$ ) the five combinations $\Upsilon(1 \mathrm{~S}) D^{0}, \Upsilon(2 \mathrm{~S}) D^{0}$, $\Upsilon(1 \mathrm{~S}) D^{+}, \Upsilon(2 \mathrm{~S}) D^{+}$and $\Upsilon(1 \mathrm{~S}) D_{s}^{+}$, using the $7 \mathrm{TeV}$ and $8 \mathrm{TeV}$ datasets [22]. The $\Upsilon$ and open charm states are required to be in the rapidity range $2.0<y<4.5$, and the $p_{\mathrm{T}}$ ranges are $p_{\mathrm{T}}<15 \mathrm{GeV}$ $\left(1<p_{\mathrm{T}}<20 \mathrm{GeV}\right)$ for $\Upsilon$ (open charm). The cross-sections and kinematic properties of these events are found to be consistent with the expectations of double-parton-scattering (DPS), where the two particles are produced in different parton-parton interactions within the same colliding protons. The effective DPS cross-section in these events is measured to be $\sigma_{\text {eff }}=18.0 \pm 1.3$ (stat) \pm 1.2 (syst) mb.

D0 has measured the simultaneous production of $\Upsilon$ mesons with $J / \psi$ candidates, in protonantiproton collisions at $1.96 \mathrm{TeV}$ [23]. The prompt component is separated using the $J / \psi$ decay length, leading to a total of 12 candidates containing prompt $J / \psi$ and $\Upsilon$ mesons, with a $3.2 \sigma$ signal significance. The kinematic properties of the events, such as the azimuthal opening angle between the mesons, are consistent with DPS, and the effective cross-section for this process is determined to be $\sigma_{\text {eff }}=2.2 \pm 0.7$ (stat.) \pm 0.9 (syst.) $\mathrm{mb}$.

For the ATLAS measurement of $J / \psi$ pair production at $8 \mathrm{TeV}$ [24], the prompt di- $J / \psi$ signal is extracted using fits to the distributions of invariant mass, decay length and longitudinal separation, and the DPS contribution is estimated using $J / \psi$ candidates taken from different events. The differential cross-section is measured as a function of the $p_{\mathrm{T}}$ of the sub-leading $J / \psi$, and is consistent with contributions from direct production as well as DPS. The effective DPS crosssection is measured as $\sigma_{\text {eff }}=8.7 \pm 1.8 \mathrm{mb}$; the range of $\sigma_{\text {eff }}$ values presented here indicates that the dependence of this parameter on the process and collision energy requires more study.

\section{Summary}

Several recent measurements of charm hadron production have been presented, including the first results from $13 \mathrm{TeV}$ collisions at LHC Run 2. These new data extend the reach to higher $p_{\mathrm{T}}$ and continue to probe QCD phenomena at greater precision.

\section{Acknowledgments}

The author was supported by a Royal Society Research Fellowship.

\section{References}

[1] ATLAS Collaboration, The ATLAS Experiment at the CERN Large Hadron Collider, JINST 3, 98 S08003 (2008).

[2] ATLAS Collaboration, Measurement of the differential cross-sections of prompt and non-prompt production of $J / \psi$ and $\psi(2 S)$ in pp collisions at $\sqrt{s}=7$ and 8 TeV with the ATLAS detector, Eur. Phys. J. C76 (2016) 283 [arXiv: 1512.03657 ]. 
[3] G. T. Bodwin, E. Braaten and G. P. Lepage, Rigorous QCD analysis of inclusive annihilation and production of heavy quarkonium, Phys. Rev. D51 (1995) 1125, [arXiv: hep-ph/9407339].

[4] M. Cacciari et al., Theoretical predictions for charm and bottom production at the LHC, JHEP 10 (2012) 137, [arXiv:1205.6344].

[5] ALICE Collaboration, Inclusive quarkonium production at forward rapidity in pp collisions at $\sqrt{\mathrm{s}}=8$ TeV, Eur. Phys. J. C76 (2016) 184 [arXiv: 1509.08258$].$

[6] LHCb Collaboration, Production of $J / \psi$ and Upsilon mesons in pp collisions at $\sqrt{s}=8 \mathrm{TeV}$, JHEP 06 (2013) 064 [arXiv: 1304.6977$].$

[7] CMS Collaboration, Quarkonium production cross sections in pp collisions at $\sqrt{s}=13 \mathrm{TeV}$, CMS Physics Analysis Summary CMS-PAS-BPH-15-005.

[8] LHCb Collaboration, Measurement of forward J/ $\psi$ production cross-sections in pp collisions at $\sqrt{s}=13 \mathrm{TeV}$, JHEP 10 (2015) 172 [arXiv: 1509.00771$]$.

[9] ATLAS Collaboration, Measurement of the differential non-prompt $J / \psi$ production fraction in $\sqrt{s}=13$ TeV pp collisions at the ATLAS experiment, ATLAS Note ATLAS-CONF-2015-030.

[10] CDF Collaboration, Measurement of the $D^{+}$-meson production cross section at low transverse momentum in $p \bar{p}$ collisions at $\sqrt{s}=1.96 \mathrm{TeV}$ [arXiv: 1610.08989 ].

[11] ATLAS Collaboration, Measurement of $D^{* \pm}, D^{ \pm}$and $D_{s}^{ \pm}$meson production cross sections in pp collisions at 7 TeV with the ATLAS detector, Nucl. Phys. B 907 (2016) 717 [arXiv: 1512.02913 ].

[12] B. A. Kniehl et al., Phys. Rev. D 71 (2005) 014018, [arXiv: hep-ph/ 0410289 ].

[13] P. Nason, A new method for combining NLO QCD with shower Monte Carlo algorithms, JHEP 11 (2004) 040, [hep-ph/ 0409146$].$

[14] S. Frixione and B. R. Webber, Matching NLO QCD computations and parton shower simulations, JHEP 06 (2002) 029, [hep-ph / 020424 4].

[15] LHCb Collaboration, Measurements of prompt charm production cross-sections in pp collisions at $\sqrt{s}=13 \mathrm{TeV}$, JHEP 03 (2016) 159, JHEP 09 (2016) 013 [arXiv : 1510 . 01707 ].

[16] ALICE Collaboration, D-meson production in $p$-Pb collisions at $\sqrt{s_{\mathrm{NN}}}=5.02$ TeV and in pp collisions at $\sqrt{s}=7 \mathrm{TeV}$ [arXiv:1605.07569].

[17] R. Maciula and A. Szczurek, Open charm production at the LHC - $k_{t}$-factorization approach, Phys. Rev. D87 9, (2013) 094022, [arXiv:arXiv: 1301 . 3033].

[18] CMS Collaboration, $\mathrm{D}^{0}$ meson nuclear modification factor in PbPb collisions at $\sqrt{s_{\mathrm{NN}}}=5.02 \mathrm{TeV}$, CMS Physics Analysis Summary CMS-PAS-HIN-16-001.

[19] LHCb Collaboration, Study of cold nuclear matter effects using prompt $\mathrm{D}^{0}$ meson production in pPb collisions at LHCb, LHCb Note LHCb-CONF-2016-003.

[20] ALICE Collaboration, Measurement of D-meson production versus multiplicity in $p$-Pb collisions at $\sqrt{\mathrm{s}_{\mathrm{NN}}}=5.02 \mathrm{TeV}$, JHEP 08 (2016) 078 [arXiv: 1602.0724 ] ; also JHEP 09 (2015) 148 [arXiv:1505.00664] for proton-proton collisions.

[21] ALICE Collaboration, Measurement of azimuthal correlations of D mesons and charged particles in pp collisions at $\sqrt{s}=7 \mathrm{TeV}$ and p-Pb collisions at $\sqrt{s_{\mathrm{NN}}}=5.02 \mathrm{TeV}$ [arXiv:1605.06963].

[22] LHCb Collaboration, Production of associated $Y$ and open charm hadrons in pp collisions at $\sqrt{s}=7$ and $8 \mathrm{TeV}$ via double parton scattering, JHEP 07 (2016) 052 [arXiv: 1510.05949 ].

[23] D0 Collaboration, Evidence for simultaneous production of $J / \psi$ and $\Upsilon$ mesons, Phys. Rev. Lett. 116 (2016) 082002 [arXiv:1511.02428].

[24] ATLAS Collaboration, Measurement of the prompt $J / \psi$ pair production cross-section in pp collisions at $\sqrt{s}=8 \mathrm{TeV}$ with the ATLAS detector, ATLAS Note ATLAS-CONF-2016-047. 